\title{
Artesunate Attenuates Pro-Inflammatory Cytokine Release from Macrophages by Inhibiting TLR4-Mediated Autophagic Activation via the TRAF6-Beclin1-PI3KC3 Pathway
}

\author{
Mei Kuang ${ }^{\text {a }}$ Yanyan Cen ${ }^{\mathrm{a}} \quad$ Rongxin Qin ${ }^{\mathrm{a}} \quad$ Shenglan Shang ${ }^{\mathrm{a}} \quad$ Zhaoxia Zhai \\ Chao Liu ${ }^{a}$ Xichun Pan ${ }^{a}$ Hong Zhou a,b
}

aDepartment of Pharmacology, College of Pharmacy, the Third Military Medical University, Chongqing, ${ }^{b}$ Key Laboratory of Basic Pharmacology of Ministry of Education and Joint International Research Laboratory of Ethnomedicine of Ministry of Education, Zunyi Medical University, Zunyi, China

\section{Key Words}

Artesunate Lipopolysaccharide • Pro-inflammatory cytokine • Autophagy • TRAF6 • Beclin1 - PI3KC3

\begin{abstract}
Background/Aims: Lipopolysaccharide (LPS) plays a critical role in excessive inflammatory cytokine production during sepsis. Previously, artesunate (AS) was reported to protect septic mice by reducing LPS-induced pro-inflammatory cytokine release. In the present study, the possible mechanism of the anti-inflammatory effect of AS was further investigated. Methods: An enzyme-linked immunosorbent assay was used to detect TNF- $\alpha$ and IL- 6 release from macrophages. Specific small interfering RNAs (siRNAs) were used to knockdown the mRNA expression of target genes. Transmission electron microscopy and laser confocal microscopy were used to observe changes in autophagy. Western blotting was performed to detect the protein levels of tumor necrosis factor receptor-associated factor6 (TRAF6), Beclin1, phosphatidylinositol 3-kinase class III (PI3KC3), autophagy-related protein 5 (ATG5), and sequestosome 1. Immunoprecipitation (IP) and fluorescent co-localization were used to detect the interactions between TRAF6-Beclin1 and Beclin1-PI3KC3, and the ubiquitination of Beclin1. Results: AS inhibited TNF- $\alpha$ and IL- 6 release from RAW264.7 cells, mouse bone marrow-derived monocytes (BMDMs) and peritoneal macrophages (PMs) induced by LPS. However, the inhibition by AS of LPS-induced cytokine release decreased when autophagy was inhibited using 3-MA, bafilomycin A1, or a siRNA targeting the Atg5 gene. Notably, AS showed an inhibition of LPS-induced autophagic activation not degradation. Whereas, these effects of AS were lost in macrophages lacking TLR4 and decreased in macrophages with down-regulated TRAF6, indicating that AS inhibited LPS-induced cytokine release and autophagic activation via TLR4-TRAF6 signaling. Western blotting results showed AS could reduce the levels of TRAF6, Beclin1, and PI3KC3. Importantly, the IP results showed AS only

Xichun Pan

and Hong Zhou

Department of Pharmacology, College of Pharmacy

The Third Military Medical University, Chongqing (China)

E-Mail zhouh64@163.com
\end{abstract}

KARGER 


\section{Cellular Physiology Cell Physiol Biochem 2018;47:475-488 \\ \begin{tabular}{l|l} 
DOI: 10.1159/000489982 & O 2018 The Author(s). Published by S. Karger AG, Basel \\
wwww.karger.com/cpb
\end{tabular} \\ Kuang et al.: Artesunate Inhibits the TRAF6-Beclin1-PI3KC3 Pathway}

inhibited K63-linked ubiquitylation not total ubiquitylation of Beclin1 by acting on TRAF6. This interrupted the TRAF6-Beclin1 interaction and subsequent the formation of Beclin1PI3KC3 core complex of the PI3K-III complex. Conclusion: AS inhibited LPS-induced cytokine release from macrophages by inhibiting autophagic activation. This effect was tightly related to blockade of the TRAF6-Beclin1-PI3KC3 pathway via decreasing K63-linked ubiquitination of Beclin1 and then interrupting the formation of Beclin1-PI3KC3 core complex of the PI3K-III complex. Our findings reveal the mechanism of AS's anti-inflammatory effect and is significant for future targeted investigations of sepsis treatment.

\section{Introduction}

Sepsis is a progressive and life-threatening clinical syndrome that is characterized by an unbalanced inflammatory and innate immune response to infection [1]. Despite improvements in supportive care, there are no effective drugs to treat sepsis and there has been no improvement in hospital mortality over the past decade [2]. Therefore, it is important to investigate new treatments with the aim of developing a clinically effective anti-sepsis drug.

The defensive responses to sepsis involve engagement of the innate immune system. This system attempts to remove invasive microorganisms and elicits a robust and acute inflammatory response [3]. In the innate immune system, monocytes and macrophages play a very important role via recognizing pathogens and producing pro-inflammatory cytokines [4].

Lipopolysaccharide/endotoxin (LPS) is well recognized pathogenic factor that induces sepsis and is thus regarded as an important drug target for sepsis [5]. Innate immune system monocytes/macrophages use toll-like receptor 4 (TLR4) to recognize LPS, which then triggers myeloid differentiation factor 88 (MyD88)-dependent or -independent activation of tumor necrosis factor receptor-associated factor6 (TRAF6), known as an E3 ubiquitin ligase. This in turn activates nuclear factor kappa B (NF- $\kappa B$ ) to promote the release of proinflammatory cytokines such as tumor necrosis factor alpha (TNF- $\alpha$ ) and interleukin 6 (IL6) [5]. Despite inducing an inflammatory response, TLR4 is also a sensor of autophagy. It promotes TRAF6-dependent K63-linked ubiquitination of Beclin1 (also named as Vps30 or Atg6) and thus activates phosphatidylinositol 3-kinase class III (PI3KC3), resulting in the activation of PI3K-III complex-mediated autophagy [6, 7]. Moreover, TLR4 triggers TRAF6 to recruit sequestosome 1 (SQSTM1/p62), thereby facilitating aggrephagy, a type of selective autophagy with aggresome-like induced structures [8]. In fact, LPS-induced autophagy and cytokine production can affect each other [9]. Th1 cytokines, such as interferon (IFN)- $\gamma$, TNF- $\alpha$, IL-6, and TGF- $\beta$, can induce autophagy [9]. Consistently, autophagy can also enhance the production of TNF- $\alpha$ and IL-6 [9].

Previously, we found that the anti-malarial compound artesunate (AS) could protect sepsis model mice challenged with heat-killed Escherichia coli [10]. The protection of AS was mainly related to reduced LPS-induced pro-inflammatory cytokines release $[10,11]$. Therefore, AS is considered as a candidate anti-sepsis drug. However, the mechanisms of the anti-inflammatory effect of AS remain unclear. Considering that autophagy plays a crucial role in LPS-induced inflammation, in the present study, we aimed to reveal the antiinflammatory mechanism of AS combined with insights into autophagy.

\section{Materials and Methods}

\section{Reagents}

LPS, 3-Methyladenine (3-MA), bafilomycin A1 (Baf), Small interfering RNAs (siRNAs) and secondary antibodies were purchased from Sigma (St. Louis, MO, USA). AS was purchased from Guilin Pharmaceuticals (Guangxi, China). Primary antibodies against Beclin1, TRAF6, PI3KC3, phospho-PI3KC3 (Ser249), LC3B, 


\section{Cellular Physiology Cell Physiol Biochem 2018;47:475-488 \begin{tabular}{l|l|l} 
DOI: 10.1159/000489982 & O 2018 The Author(s). Published by S. Karger AG, Basel \\
www.karger.com/cpb
\end{tabular} \\ Kuang et al.: Artesunate Inhibits the TRAF6-Beclin1-PI3KC3 Pathway}

ATG5, SQSTM1, total ubiquitin, and K63-linked ubiquitin were purchased from Cell Signaling (Beverly, MA, USA). SuperSignal chemiluminescent substrate, the RFP-GFP-LC3B Kit, Lipofectamine2000, and Pierce Protein A/G Magnetic Beads were obtained from Thermo Fisher (Waltham, MA, USA). Mouse TNF- $\alpha$ and IL-6 enzyme-linked immunosorbent assay (ELISA) kits were obtained from Affymetrix (San Diego, CA, USA). Dulbecco's modified Eagle's medium (DMEM) and fetal bovine serum (FBS) were obtained from Gibco (Grand Island, NY, USA).

Animals and Cell lines

Specific-pathogen-free KM mice and TLR4\% C57 mice (4-6 weeks old, weighing 18-22 g) were obtained from the Experimental Animal Center of our university. Peritoneal macrophages (PMs) and bone marrow-derived macrophages (BMDMs) were isolated and cultured as described previously [12, 13]. All animal experiments were performed according to the National Guidelines for Animal Care and Use. The macrophage cell line RAW264.7 was obtained from ATCC (Manassas, VA, USA). Cells were cultured in DMEM with $10 \%(\mathrm{v} / \mathrm{v}) \mathrm{FBS}$ at $37{ }^{\circ} \mathrm{C}$ in $5 \% \mathrm{CO}_{2}$. Before treatment, the medium was replaced with fresh medium without FBS.

\section{ELISA}

RAW264.7 cells, PMs, or BMDMs grown in 96-well plates $\left(2 \times 10^{5}\right.$ cells/well) were treated with AS, 3-MA, Baf, and LPS, as indicated. The supernatants were collected to detect TNF- $\alpha$ and IL- 6 concentrations using their respective ELISA kits, according to the manufacturers' protocols.

\section{Western Blotting}

Equal amounts of protein lysates extracted from RAW264.7 cells were separated by SDS-PAGE. The protein bands were then transferred onto polyvinylidene fluoride (PVDF) membranes, blocked with $5 \%$ skim milk, probed with the designated primary antibodies and secondary horseradish peroxidase (HRP)-conjugated antibodies. Chemiluminescence-marked images were developed using the SuperSignal chemiluminescent substrate with a ChemiDoc ${ }^{\mathrm{TM}}$ Touch Imaging System (Bio-Rad, Hercules, CA, USA) and analyzed using the ImageLab packages (Bio-Rad).

\section{Autophagy assays}

To observe autophagosome-like vesicles, RAW264.7 cells grown in dishes were pre-treated with AS (20 $\mu \mathrm{g} / \mathrm{mL}$ ) for $2 \mathrm{~h}$ followed by LPS (100 ng/mL) for $1 \mathrm{~h}$. Cells were harvested to observe their ultrastructure using a transmission electron microscope (JEM-1400PLUS; JEOL Ltd, Tokyo, Japan) at $60 \mathrm{kV}$, according to a previous report [14]. To detect LC3 dots formation, RAW264.7 cells or BMDMs grown on glass slides were treated as indicated. The cells were then fixed with paraformaldehyde (4\%; m/v) for $10 \mathrm{~min}$, blocked with phosphate-buffered saline (PBS; $50 \mu \mathrm{M} ; \mathrm{pH}$ 7.2-7.4) containing bovine serum albumin (BSA; 1\%, $\mathrm{m} / \mathrm{v}$ ) and Triton X-100 (0.1\%; m/v) for $20 \mathrm{~min}$, probed with anti-LC3B antibody overnight followed by secondary antibodies conjugated with Alexa Fluor 488 for 2 h. Then nuclei were stained with 4, 6-diamidino-2phenylindole dihydrochloride (DAPI). LC3 dots (green) were observed using a Zeiss LSM780 confocal microscopy (Jena, Germany). To detect the autophagic flux, RAW264.7 cells grown on glass slides were transfected using a RFP-GFP-LC3B kit, according to the manufacturer's handbook, and then treated with AS and LPS as indicated. After fixation and staining the nuclei, autophagosomes (yellow) and autolysosomes (red) were observed using confocal imaging, and quantified using the ImageJ packages.

\section{RNA interference}

Gene silencing was performed using specific siRNAs with a control siRNA. In brief, RAW264.7 cells were cultured to $70 \%$ confluence, and then transfected with siRNAs for $8 \mathrm{~h}$ using Lipofectamine2000, according to the manufacturer's manuals. After $16 \mathrm{~h}$ of renewed culture in complete DMEM, the cells were used for further treatment.

\section{Co-localization}

RAW264.7 cells grown on glass slides were treated with AS $(20 \mu \mathrm{g} / \mathrm{mL})$ for $2 \mathrm{~h}$, followed by LPS $(100 \mathrm{ng} / \mathrm{mL})$ for $1 \mathrm{~h}$, and then fixed and blocked. Beclin 1 was probed using mouse anti-Beclin 1 antibody overnight, followed by Alexa Fluor 488-conjugated secondary antibody for $2 \mathrm{~h}$. TRAF6 or PI3KC3 was 


\section{Cellular Physiology Cell Physiol Biochem 2018;47:475-488 \begin{tabular}{l|l} 
DOI: 10.1159/000489982 & O 2018 The Author(s). Published by S. Karger AG, Basel \\
www.karger.com/cpb
\end{tabular} \\ Kuang et al.: Artesunate Inhibits the TRAF6-Beclin1-PI3KC3 Pathway}

probed using corresponding rabbit primary antibodies overnight, followed by Alexa Fluor 555-conjugated secondary antibody for $2 \mathrm{~h}$. The nuclei were stained with DAPI. Co-localization of Alexa Fluor 488 (green) and Alexa Fluor 555 (red) was observed using confocal imaging. The Pearson's correlation coefficients of Beclin1 and TRAF6, or Beclin1 and PI3KC3 were calculated using the ImageJ packages.

Immunoprecipitation

3'-Flag tagged Traf6 (GenBank No. NM_001303273.1) and 3'-Flag tagged Beclin1 (NM_019584) were synthesized and sub-cloned into vector pcDNA3.1 (Thermo Fisher), separately. The constructs were verified by DNA sequencing. Immunoprecipitation (IP) was performed according to the manual of the Protein A/G Magnetic Beads kit. RAW264.7 cells grown in 10-cm dishes were transfected with the flag-Traf6 or flagBeclin1 constructs using Lipofectamine2000, according to the manufacturer's instructions. After treatment as indicated, the whole cell lysate was extracted using the cell lysis-wash buffer supplied in the kit, and $1 \mathrm{mg}$ of proteins were incubated with primary antibodies at $4{ }^{\circ} \mathrm{C}$ overnight. Immune complexes were collected using the Protein A/G beads and subjected to SDS-PAGE and western blotting as described above.

\section{Statistics}

Cytokine concentrations and other data in charts are shown as the means \pm SD. Student's $t$-test was used for pairwise comparisons. Differences with a $P$ value less than 0.05 were considered statistically significant, and those less than 0.01 were considered highly statistically significant.

\section{Results}

\section{AS inhibits LPS-induced cytokine release from macrophages}

LPS activates TLR4 signaling, leading to the release of TNF- $\alpha$ and IL-6, and subsequent inflammation [15]. Our previous report showed that AS could inhibit LPS-induced proinflammatory cytokine release in vivo and in vitro [11]. In the present study, the inhibition by AS of LPS-stimulated TNF- $\alpha$ and IL-6 release from mouse RAW264.7 cells, PMs, and BMDMs were further confirmed. Based on the results of the dose-effect relationship of LPS and the cytokines (Fig. 1A, B), an LPS concentration of $100 \mathrm{ng} / \mathrm{mL}$ was selected for subsequent experiments.

Although AS alone had no influence on the release of TNF- $\alpha$ and IL-6, AS dosedependently decreased LPS-induced cytokines release from RAW264.7 cells (Fig.1C, 1D). For the RAW264.7 cell line, the inhibition by AS $(20 \mu \mathrm{g} / \mathrm{mL})$ of TNF- $\alpha$ and IL- 6 release was $56.0 \%$ and $71.4 \%$, respectively. Similar results were obtained using primary mouse macrophages, including BMDMs and PMs. In BMDMs, the inhibition by AS of TNF- $\alpha$ and IL- 6 release was $35.3 \%$ and $48.7 \%$, respectively (Fig. 1E, 1F). In PMs, the inhibition by AS of TNF- $\alpha$ and IL- 6 release was $66.4 \%$ and $65.4 \%$, respectively (Fig. $1 \mathrm{G}, 1 \mathrm{H}$ ). These results demonstrated that AS has a significant anti-inflammation effect.

\section{AS cannot exert its anti-inflammation effect if autophagy is inhibited}

Autophagy is considered an important component of the innate immune response. LPS can induce autophagy, and autophagy participates in LPS-induced inflammation [16]. Therefore, several autophagy inhibitors including 3-MA, Baf, and siRNA targeting the autophagy-related protein 5 gene (Atg5) were used to evaluate whether AS's antiinflammatory effect was related to autophagy.

First, the autophagy inhibitor 3-MA, which targets PI3 KC3, was used. The results showed that 3-MA markedly inhibited LPS-induced TNF- $\alpha$ and IL-6 release, to a greater extent than AS. However, there was no synergy between the 3-MA and AS (Fig. 2A, 2B), suggesting that autophagy contributes to LPS-induced cytokine production and AS's anti-inflammatory effect. Second, another autophagy inhibitor Baf, which targets lysosome acidification and blocks autophagic flux, presented similar inhibition of LPS-induced TNF- $\alpha$ and IL- 6 release to 3-MA (Fig. 2C, 2D). Finally, siRNA targeting Atg5, encoding a key molecule for autophagy [17], was used. The results showed that knockdown of Atg 5 led to almost no TNF- $\alpha$ and IL-6 release. Unsurprisingly, AS also lost its effect in the presence of the siRNA (Fig.2E, 2F). The 


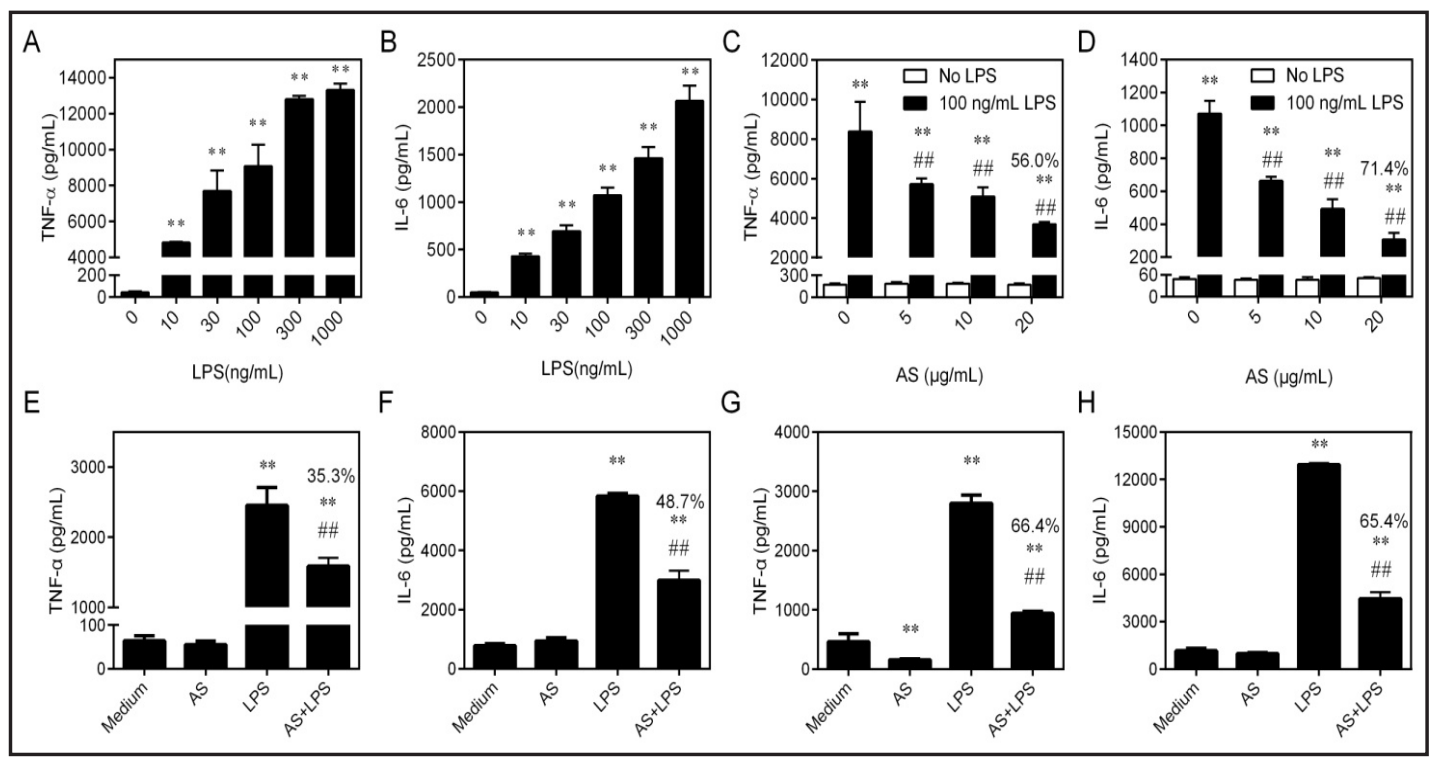

Fig. 1. AS inhibits LPS-induced cytokine release from macrophages. (A, B) RAW264.7 cells grown in 96-well plates were treated with LPS $(0,10,30,100,300$, and $1000 \mathrm{ng} / \mathrm{mL})$ at $37^{\circ} \mathrm{C}$ for $6 \mathrm{~h}$. The supernatants were collected to detect TNF- $\alpha$ and IL- 6 using ELISA. (C, D) RAW264.7 cells were pre-treated with AS (0, 5, 10, and $20 \mu \mathrm{g} / \mathrm{mL}$ ) at $37^{\circ} \mathrm{C}$ for $2 \mathrm{~h}$, followed by LPS (100 $\left.\mathrm{ng} / \mathrm{mL}\right)$ for $6 \mathrm{~h}$. The supernatants were collected for ELISA. (E, F) Mouse BMDMs and (G, H) PMs were pre-treated with AS $(20 \mu \mathrm{g} / \mathrm{mL})$ for $2 \mathrm{~h}$ followed by LPS (100 ng/ $\mathrm{mL}$ ) for $6 \mathrm{~h}$. The supernatants were collected for ELISA. Data from one of three independent experiments are shown $(n=4)$. The percent on the columns indicates inhibitory ratios compared with the LPS group. ${ }^{* *}$ $\mathrm{P}<0.01$ versus medium, ${ }^{\# \#} \mathrm{P}<0.01$ versus LPS.

Fig. 2. Autophagy plays a crucial role in the anti-inflammatory effect of AS. (A, B) RAW264.7 cells grown in 96well plates were pre-treated with AS $(20 \mu \mathrm{g} / \mathrm{mL})$ or $3-\mathrm{MA}$ (4 mM) for $2 \mathrm{~h}$, followed by LPS (100 ng/mL) for $6 \mathrm{~h}$. The supernatants were collected for ELISA. (C, D) Experiments were performed as shown in A. Cells were pre-treated with AS $(20 \mu \mathrm{g} / \mathrm{mL})$ or Baf (10 ng/mL) for 2 h. (E, F) Experiments were performed as shown in A. Cells were transfected with an Atg5 siRNA or control siRNA for $6 \mathrm{~h}$, and then culture was renewed for $24 \mathrm{~h}$. Data from one of three independent experiments are shown $(\mathrm{n}=4) . * * \mathrm{P}<0.01, *$ $\mathrm{P}<0.05, \S \mathrm{P}>0.05$.

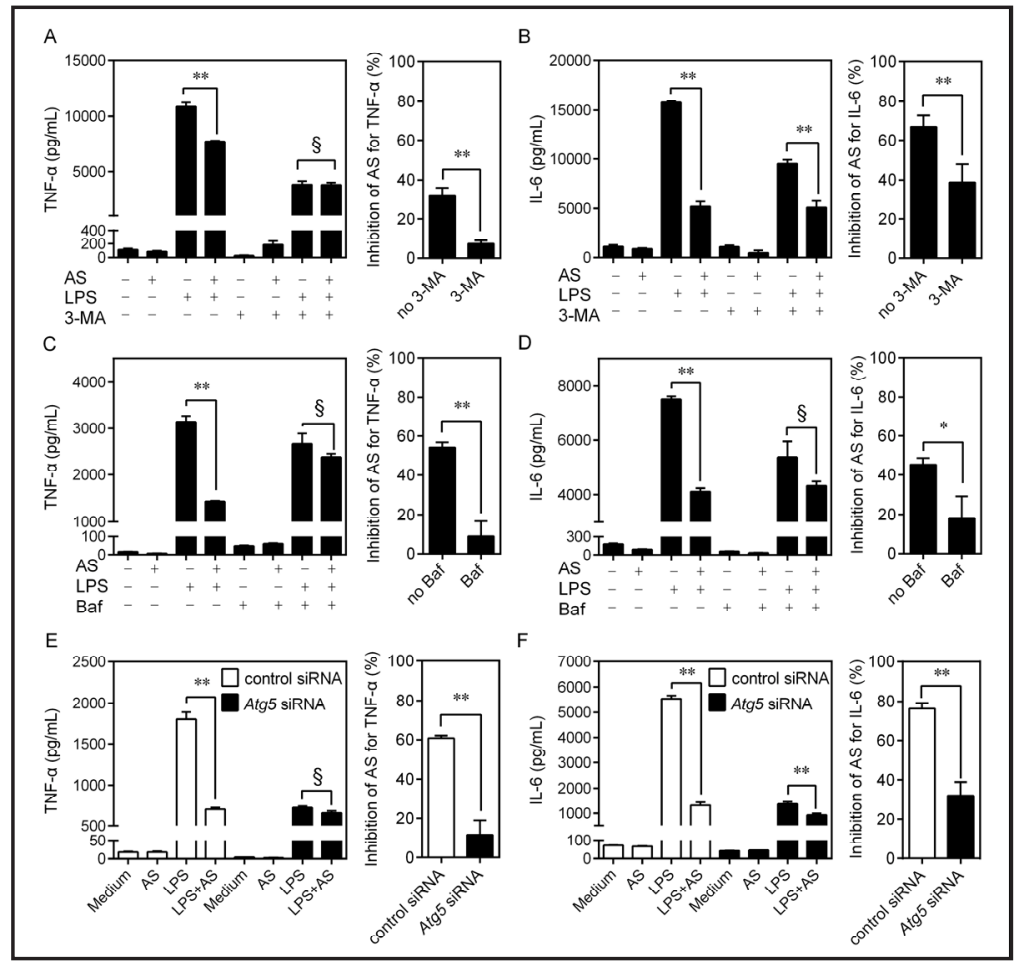

above results demonstrated that autophagy indeed participates in LPS-induced cytokine production, and that AS cannot exert its anti-inflammation effect if autophagy is inhibited.

\section{KARGER}




\section{Cellular Physiology Cell Physiol Biochem 2018;47:475-488

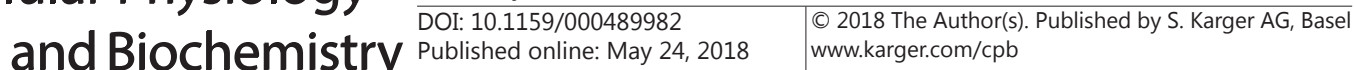 \\ Kuang et al.: Artesunate Inhibits the TRAF6-Beclin1-PI3KC3 Pathway}

AS inhibits LPS-stimulated autophagic activation of macrophages

The above results showed that autophagy contributes to LPS-induced cytokine production and AS's anti-inflammation effect; therefore, the influence of AS on autophagyrelated molecules was tested, and markers such as the conversion of LC3B-I to LC3B-II, the accumulation of ATG5 and degradation of sequestosome 1 (SQSTM1), were selected to evaluate autophagy activity [18]. Western blotting showed that LPS induced an increase in LC3B-II and ATG5 levels for up to $1 \mathrm{~h}$, and their levels began to decrease after $2 \mathrm{~h}$ in both RAW264.7 cells and BMDMs (Fig. 3A). However, SQSTM1 levels decreased after $0.5 \mathrm{~h}$ and were restored from $16 \mathrm{~h}$ in RAW264.7 and $4 \mathrm{~h}$ in BMDMs. Therefore, $1 \mathrm{~h}$ was selected as the best time-point to observe LPS-induced autophagic activation.

Transmission electron microscopy (TEM) observation showed that although AS could increase the number of autophagic vesicles within cells compared with those in the control, LPS had a more potent effect. However, AS could significantly inhibit the formation of autophagic vesicles induced by LPS (Fig. 3B).

Consistently, there was a marked increase in amount of LC3 dots in RAW264.7 or BMDM cells treated with LPS alone, while AS significantly decreased the amount of LPS-induced LC3 dots (Fig. 3C). Furthermore, western blotting showed that AS also inhibited LPS-induced accumulation of LC3B-II and ATG5, but had no influence on SQSTM1 degradation within both RAW264.7 cells and BMDMs (Fig. 3D). The above findings suggested that AS inhibits LPSinduced autophagosome formation by inhibiting autophagic activation but not autophagic degradation.

To further determine whether the effects of AS are related to the inhibition of autophagic activation, RAW264.7 cells were transfected with a pH-sensitive tandem RFPGFP-LC3B construct and the co-localization of GFP-LC3 and RFP-LC3 dots were observed after treatment with LPS and AS. The results showed that LPS greatly increased the amount of autophagosomes (GFP and RFP co-localization) and autolysosomes (RFP). However, AS significantly inhibited the LPS-induced accumulation of autophagosomes, but not autolysosomes (Fig. 3E, 3F), again demonstrating that AS inhibits LPS-induced autophagic activation.

Furthermore, the influence of AS on LPS-induced LC3 dots formation and LC3B-II accumulation in RAW264.7 cells were examined using the Atg5 siRNA. The Atg5 siRNA decreased number of LC3 dots and LC3B-II accumulation in RAW264.7 cells treated with LPS; however, AS lost its inhibition of LPS-induced LC3 dots formation and LC3B-II accumulation in cells treated with the Atg 5 siRNA (Fig. 3G, 3H). These results confirmed that AS inhibits LPS-induced autophagic activation of macrophages.

TLR4 is required for AS's inhibition of LPS-induced cytokines release and autophagic activation

TLR4 is the receptor for LPS recognition and is the sensor for autophagy induced by LPS [16]. To determine whether the inhibition by AS of LPS-induced cytokines release and autophagic activation is related to TLR4, BMDMs lacking TLR4 (TLR4 ${ }^{--}$) were tested. In terms of cytokines release, the results showed that LPS could significantly induce TNF- $\alpha$ and IL-6 release from wildtype cells, but not from the cells lacking TLR4 (TLR4 $\%$ ) (Fig. 4A). Notably, AS could significantly inhibit TNF- $\alpha$ and IL-6 release from wildtype cells but showed no effect on their release from cells lacking TLR4 (Fig. 4A). In terms of autophagic activation, LPS had a strong effect on LC3 dots formation and the levels of ATG5 and LC3B-II in wildtype cells, but had no effect in the cells lacking TLR4 (Fig. 4B - 4D). Unsurprisingly, AS's inhibition of LPS-induced autophagic activation was observed in wildtype cells but not in cells lacking TLR4 (Fig. 4B-4D). These findings demonstrated that AS's inhibition of LPSinduced cytokines release and autophagy is closely related to TLR4 activity, and TLR4 is required for AS's effect. 


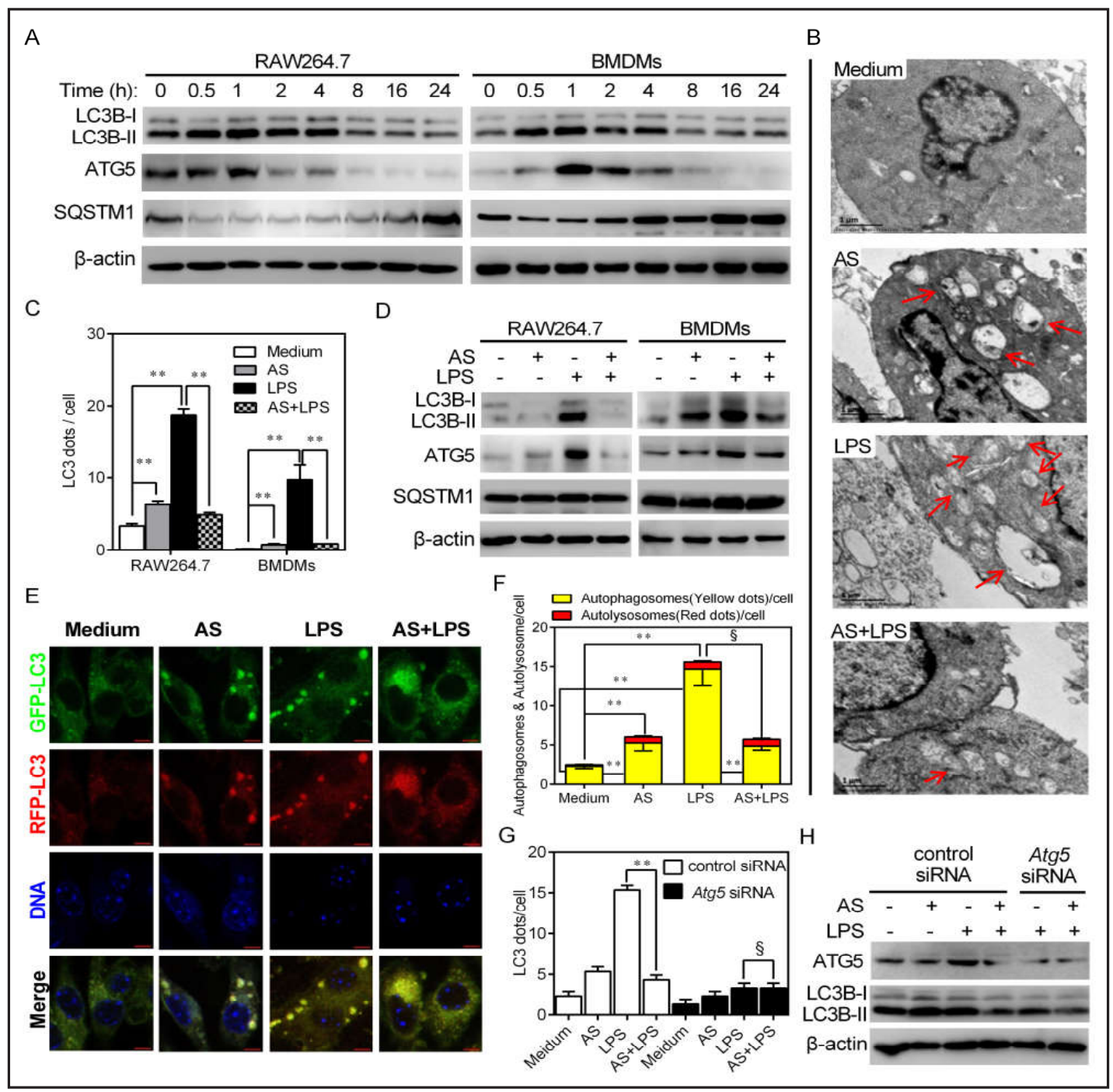

Fig. 3. AS inhibits LPS-stimulated autophagic activation of macrophages. (A) RAW264.7 cells or mouse BMDMs were treated with LPS (100 ng/mL) for up to $24 \mathrm{~h}$. The levels of LC3B-I/II, ATG5, and SQSTM1 were determined by western blotting. (B) RAW264.7 cells were treated with AS ( $20 \mu \mathrm{g} / \mathrm{mL})$ for $2 \mathrm{~h}$ followed by LPS (100 ng/mL) for $1 \mathrm{~h}$. Cells were collected to observe their ultrastructure by TEM. The arrows indicate autophagosome-like vesicles. (C) RAW264.7 cells or mouse BMDMs grown on glass bottom dishes were treated as shown in B, fixed, stained with DAPI (blue), and probed with anti-LC3 antibody, followed by Alexa Fluor 488-conjugated goat anti-rabbit IgG (green). The LC3 dots were observed using confocal microscopy and counted using Image $(n=50)$. (D) RAW264.7 cells or mouse BMDMs were treated as shown in B. Western blotting was carried out as shown in A. (E, F) RAW264.7 cells were transfected with an RFP-GFP-LC3B kit and treated as described in B. Autophagosomes (yellow) and autolysosomes (red) were observed and counted as shown in $C(n=50$; Bar $=5 \mu \mathrm{m})$. (G) RAW264.7 cells were transfected with a control siRNA or an Atg5 siRNA. Cells were treated and observed as shown in C. (H) RAW264.7 cells were treated as shown in $\mathrm{G}$ and detected as shown in A. Data from one of three independent experiments are shown. ${ }^{* *} \mathrm{P}<0.01, \S$ $\mathrm{P}>0.05$.

TRAF6 is required for AS's inhibition of autophagic activation, but is not completely required for its inhibition of cytokines release

TRAF6 plays a key role in LPS/TLR4-induced cytokine production and Beclin1-PI3KC3mediated autophagy $[19,20]$. Therefore, the role of TRAF6 is investigated to determine whether the effects of AS are related to TRAF6 activity. We used a siRNA to downregulate 
Fig. 4. TLR4 is required for AS's inhibition of LPS-induced cytokine release and autophagic activation. (A) BMDMs isolated from wildtype C57 mice (WT) and TLR4knock-out C57 mice (TLR4\%) were treated with AS and LPS, and TNF- $\alpha$ and IL- 6 release was detected as shown in Fig. 1E ( $\mathrm{n}=$ 4). ${ }^{* *} \mathrm{P}<0.01$ versus medium, ${ }^{\# \#} \mathrm{P}<0.01$ and $\S \mathrm{P}>0.05$ versus LPS. (B, C) BMDMs from WT and TLR4\% were treated, and LC3 dots
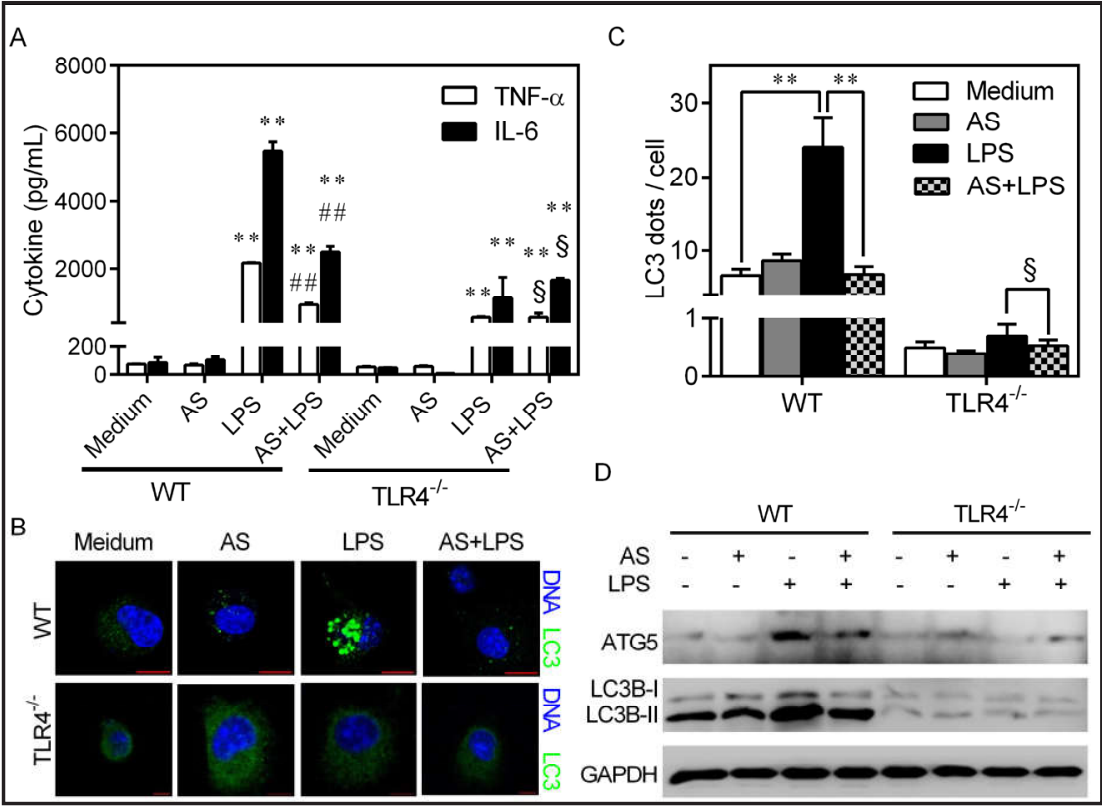

C

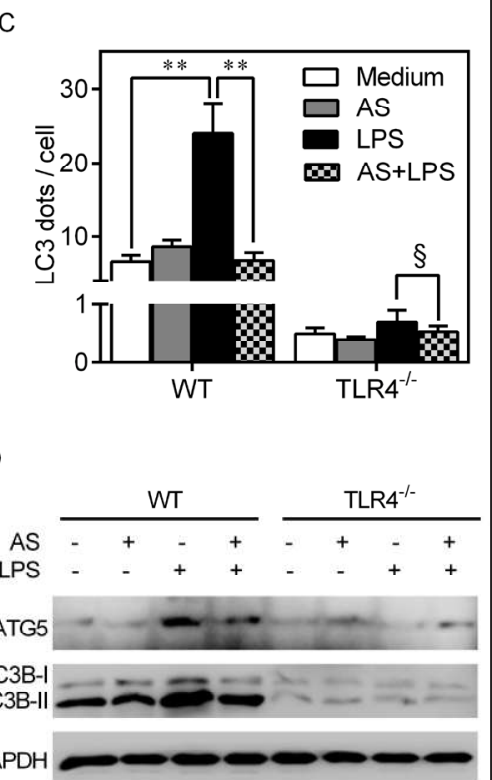

. formation was detected and measured as shown in Fig. 3C (Bar $=5 \mu \mathrm{m} ; \mathrm{n}=50)$. ${ }^{* *} \mathrm{P}<0.01, \S \mathrm{P}>0.05$. (D) BMDMs from WT and TLR4 $\%$ were treated, and the levels of ATG5 and LC3B-I/II were detected as shown in Fig. 3D. Data shown are one of three independent experiments.

Fig. 5. TRAF6 is required for AS's inhibition of LPS-induced cytokine release and autophagic activation. (A) Experiments were performed as shown in Fig. 1E. RAW264.7 cells were transfected with control siRNA or a Traf6 siRNA ( $\mathrm{n}=4$ ). ${ }^{* *} \mathrm{P}<0.01$ versus medium, \#\# $\mathrm{P}<0.01$ and ${ }^{\#} \mathrm{P}<0.05$ versus LPS. (B) Inhibition ratios of AS for cytokine release. $* \mathrm{P}<0.05$ and $* * \mathrm{P}<0.01$ versus control siRNA. (C, D) RAW264.7 cells were

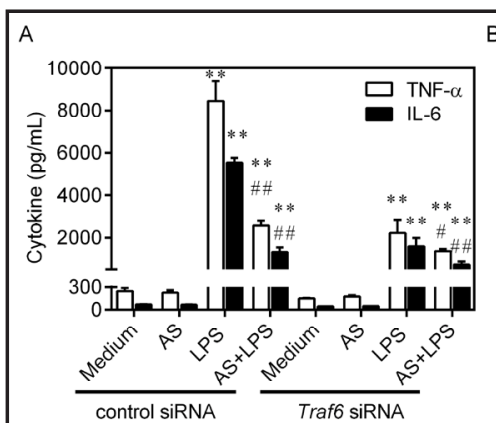
C

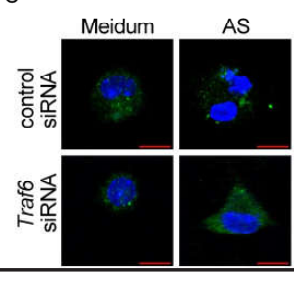
transfected with control siRNA or a Traf6 siRNA, then treated and LC3 dots formation was detected as shown in Fig. 3C $($ Bar $=5 \mu \mathrm{m})$. ** $\mathrm{P}<0.01, \S \mathrm{P}>0.05$. (E) RAW264.7 cells were transfected with control siRNA or the Traf6 siRNA. Cells were then treated, and the levels of TRAF6, ATG5 and LC3B-I/II were detected as shown in Fig. 3D. Data from one of three independent experiments are shown.

Traf6 mRNA expression. For cytokines release, the results showed that LPS could significantly induce TNF- $\alpha$ and IL- 6 release from the cells without Traf6 knockdown, but not from the cells with Traf6 knockdown. Although AS's inhibition of cytokines release was inhibited in cells with Traf6 knockdown, there was still difference in cytokines levels between the cells treated 


\section{\begin{tabular}{lll} 
Cellular Physiology & \multicolumn{1}{c}{ Cell Physiol Biochem 2018;47:475-488 } \\
DOI: 10.1159/000489982 & 0 2018 The Author(s). Published by S. Karger AG, Basel
\end{tabular}

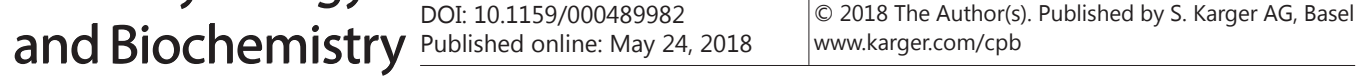

with AS and the untreated cells (Fig. 5A, 5B), which suggested that TRAF6 is important, but not completely required, for LPS-induced cytokines release and the effects of AS.

For autophagic activation, LPS had a strong effect on inducing LC3 dots formation and the levels of TRAF6 and LC3B-II in cells without Traf6 knockdown, but had no effect in cells with Traf6 knockdown (Fig. 5C - 5E). In addition, there was no difference in LC3 dots formation and LC3B-II accumulation between the cells treated with AS and the untreated cells (Fig. 5C - 5E), suggesting that TRAF6 is strictly required for LPS-induced autophagic activation and the effect of AS.

AS inhibits the levels of key molecules of the LPS-induced TRAF6-Beclin1-PI3KC3 pathway

The TRAF6-Beclin1-PI3KC3 pathway is required for LPS-induced autophagic activation [6]. The influence of AS on this pathway was investigated to determine whether AS's inhibition of LPS-induced autophagic activation was associated with this pathway.

Western blotting showed there were different changes in TRAF6, Beclin1, and total PI3KC3 or phosphorylated (Ser249)-PI3KC3 levels over $24 \mathrm{~h}$ in RAW264.7 cells and BMDMs (Fig. 6A); therefore, the time-point of $1 \mathrm{~h}$ was selected to observe the levels of these three molecules. At this time-point, LPS could significantly increase the levels of TRAF6, Beclin1, and total PI3KC3 or phosphorylated (Ser249)-PI3KC3, and AS could significantly decrease the levels of these molecules (Fig. 6B). However, in the cells with Traf6 knockdown, not only LPS, but also AS, had no effects on the levels of these molecules (Fig. 6C), which suggested that the TRAF6-Beclin1-PI3KC3 pathway is very important for the effect of AS, and that AS exerts its effects by downregulating this pathway.

AS inhibits K63-linked ubiquitylation of Beclin1 by acting on TRAF6 to interrupt the TRAF6-Beclin1 interaction

LPS induces autophagy via the TRAF6-Beclin1-PI3KC3 pathway. In this pathway, TRAF6 promotes autophagy via ubiquitinating Beclin1 (TRAF6 is an E3 ubiquitin ligase), resulting in K63-linked ubiquitination of Beclin1 [20]. To determine whether AS's inhibition of LPSinduced autophagic activation is related to its influence on ubiquitination, IP and fluorescent co-localization experiments were performed.

First, IP was performed to determine whether AS affected the TRAF6-Beclin1 interaction, which is presumed to be required for Beclin1 ubiquitination. When an equal amount of Beclin1 was used as a control (pull-down by Beclin1), AS and LPS alone, or in combination,

Fig. 6. AS downregulates the TRAF6-Beclin1-PI3KC3 pathway. (A) Experiments were performed as shown in Fig. 3A. The levels of TRAF6, Beclin1, PI3KC3, and PPI3KC3 (Ser249) in RAW264.7 and BMDMs were detected using western blotting. (B) RAW264.7 cells or BMDMs were treated as shown in Fig. 3B. TRAF6, Beclin1, PI3KC3, and P-PI3KC3 (Ser249) were detected by western blotting. (C) Experiments were performed as shown in Fig. 5E. The levels of TRAF6, Beclin1, PI3KC3, and P-PI3KC3 (Ser249) were detected by western blotting. Data shown are from one of three independent experiments.* indicates a non-specific protein band.

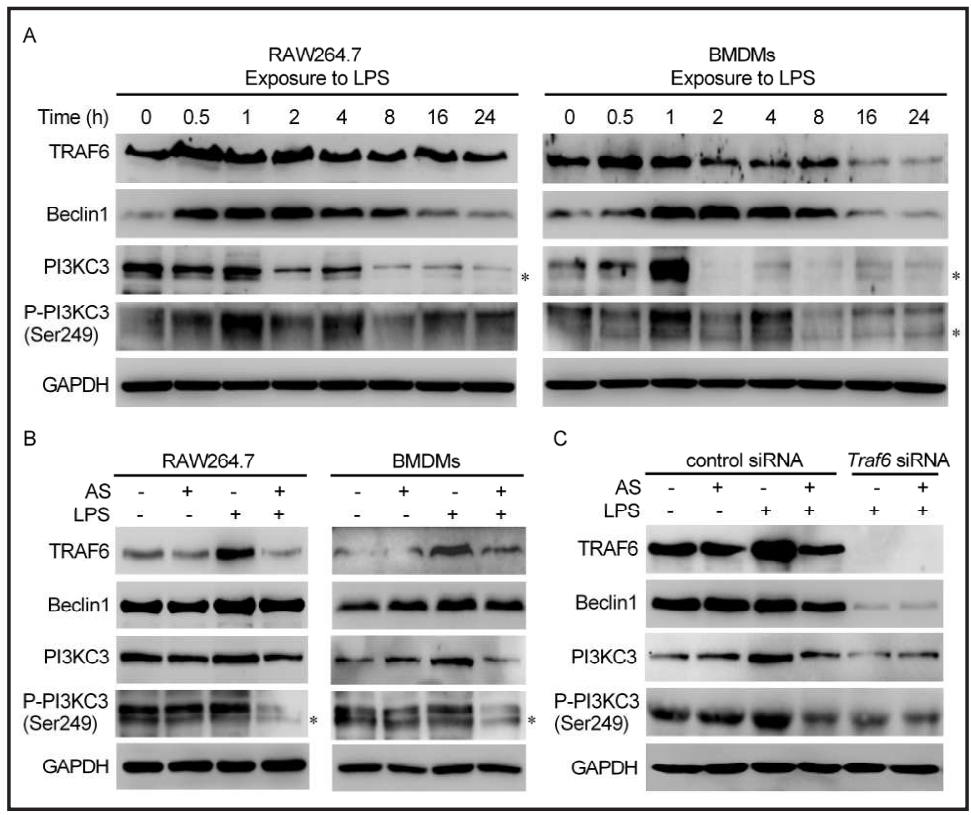




\section{Cellular Physiology Cell Physiol Biochem 2018;47:475-488 \begin{tabular}{cc|c} 
DOI: 10.1159/000489982 & $\begin{array}{l}\text { O } 2018 \text { The Author(s). Published by S. Karger AG, Basel } \\
\text { www.karger.com/cpb }\end{array}$
\end{tabular}

had no influence on the protein level of the associated TRAF6 (Fig. 7A). However, when an equal amount of TRAF6 was used as a control (pull-down by TRAF6), the protein level of the associated Beclin 1 was significantly increased by LPS, but significantly decreased by AS (Fig. 7B). These results suggested that AS might act on TRAF6 to interrupt the TRAF6-Beclin1 interaction, and further suggested that AS might inhibit the E3 ligase activity of TRAF6.

Subsequently, laser confocal microscopy imaging was used to determine whether AS affected their intracellular co-localization and distribution. LPS markedly induced colocalization and dots formation of TRAF6 and Beclin1. However, the combination of LPS with AS only significantly inhibited their co-localization, but not the induction of dots dispersion (Fig. 7C, 7D), which suggested AS only inhibited the co-localization of TRAF6 and Beclin1.

Lastly, an IP experiment was performed to detect Belcin1-associated ubiquitination using Beclin1 as an internal control. The results showed that AS and LPS, alone or in combination, had no influence on the total ubiquitination of Beclin1. Interestingly, K63linked ubiquitination of Beclin1 was markedly increased by AS or LPS alone, but significantly inhibited by AS combined with LPS (Fig. 7E), indicating that AS inhibits LPS-induced K63linked ubiquitination of Beclin1. Taken together, these results suggested that AS inhibits K63linked ubiquitination of Beclin1 by acting on TRAF6 to interrupt TRAF6-Beclin1 interaction.

\section{AS inhibits the Beclin1-PI3KC3 interaction}

Ubiquitination of Beclin1 induces autophagy by promoting the formation of the Beclin1-PI3KC3 core complex, which plays a central role in the PI3K-III complex [7]. To

Fig. 7. AS inhibits K63linked ubiquitylation of Beclin1 via TRAF6 to interrupt the TRAF6Beclin1 interaction. (A) RAW264.7 cells transfected with a flag-Beclin1 construct were treated as in Fig. 3B. The whole cell lysate was used for an IP experiment using antiflag antibody, and the associated TRAF6 was detected by immunoblotting (IB). (B) The experiment was performed as described in A. Cells were transfected with a flag-Traf6 construct, and TRAF6-associated Beclin1 was detected using immunoblotting. (C, D) The experiment was performed as described in Fig. 3C using RAW264.7
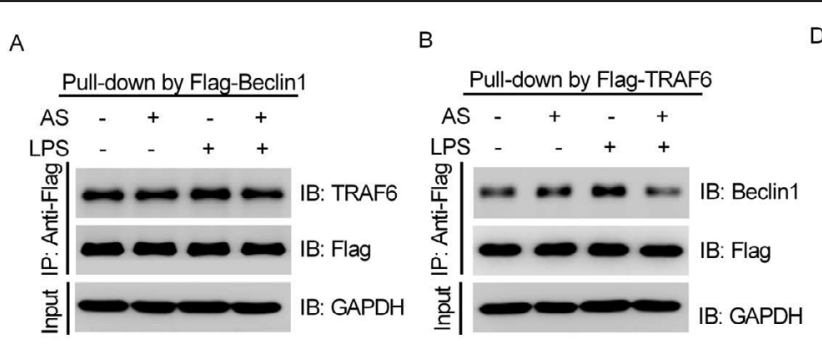

C

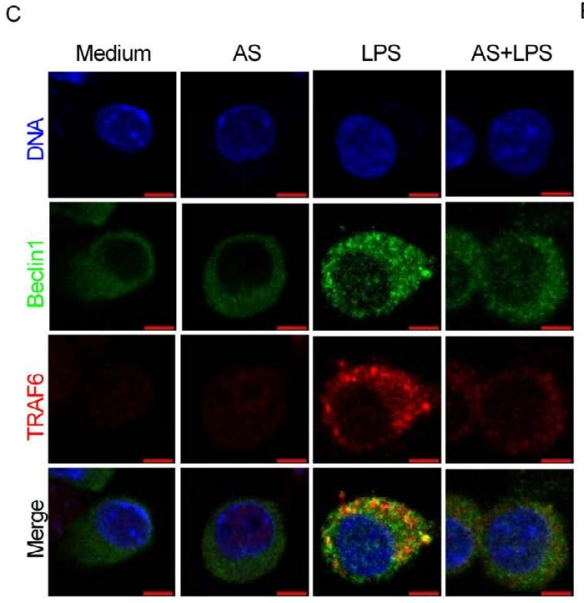

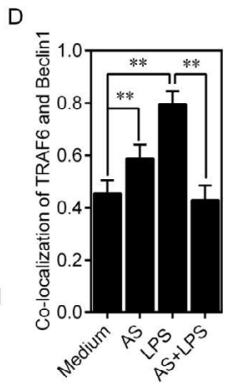

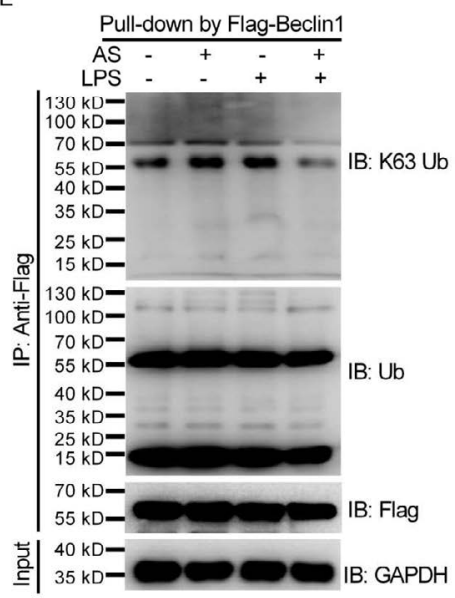
cells. Beclin1 was probed using mouse anti-Beclin1 antibody, followed by Alexa Fluor 488 (green)-conjugated goat anti-mouse IgG. TRAF6 was probed using rabbit anti-TRAF6 antibody, followed by Alexa Fluor 555 (red)-conjugated goat anti-rabbit IgG. The co-localization of Beclin1 and TRAF6 was observed using confocal imaging, and calculated using Pearson's correlation coefficient via ImageJ. (Bar $=5 \mu \mathrm{m} ; \mathrm{n}=50$; ${ }^{* *} \mathrm{P}<0.01$ ). (E) Pull-down products from A were subjected to Beclin1-associated total ubiquitin (Ub) and K63-linked ubiquitin (K63-Ub) detection using immunoblotting. 


\section{Cellular Physiology Cell Physiol Biochem 2018;47:475-488 \begin{tabular}{c|c} 
DOI: 10.1159/000489982 & O 2018 The Author(s). Published by S. Karger AG, Basel \\
wwww.karger.com/cpb
\end{tabular}

Fig. 8. AS inhibits the Bec l in 1 - P I 3 KC 3 interaction. Pull-down products from Fig. 7A were subjected to detection for the associated PI3KC3 using immunoblotting. * indicates a nonspecific band. (B) RAW264.7 cells

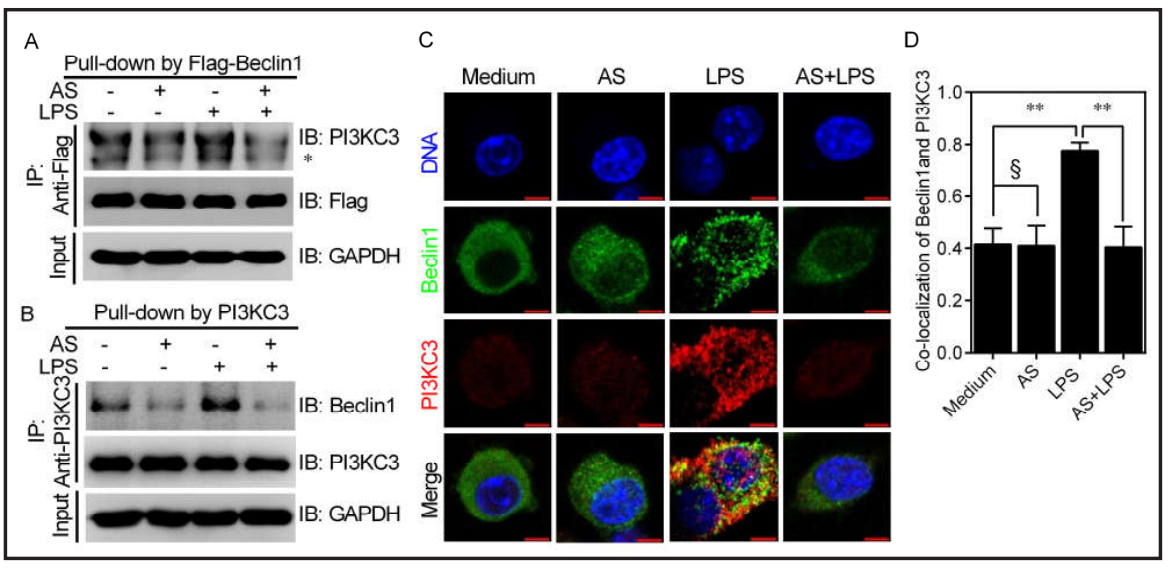
were treated as

Fig. 3B. An IP experiment was performed as shown in Fig. 7A using anti-PI3KC3 antibody, and the associated Beclin1 was detected by immunoblotting. (C, D) The experiment was performed as shown in Fig. 7C. Beclin1 was probed using Alexa Fluor 488 (green). PI3KC3 was probed using Alexa Fluor 555 (red). The co-localization of Beclin1 and PI3KC3 was observed and calculated as shown in Fig. 7C. Bar $=5 \mu \mathrm{m} ; \mathrm{n}=50$; $* * \mathrm{P}<0.01 ; \S \mathrm{P}>0.05$.

detect whether AS affects the Beclin1-PI3KC3 interaction, IP experiments were performed. When an equal amount of Beclin1 was used as control (pull-down by Beclin1), LPS increased the protein level of associated PI3KC3; however, AS decreased the level of PI3KC3 (Fig. 8A). When an equal amount of PI3KC3 was used as control (pull-down by PI3KC3), LPS increased the protein level of associated Beclin1, but AS decreased the level (Fig. 8B).

Subsequently, a laser confocal microscopy experiment was used to determine whether AS affected the co-localization and distribution of Beclin1 and PI3KC3. LPS increased the co-localization and dots formation of Beclin1 and PI3KC3. However, AS only significantly inhibited their co-localization and did not induce fluorescence dispersion (Fig. 8C, 8D). These results suggested that AS's inhibition on K63-linked ubiquitylation of Beclin1 might decrease the Beclin1-PI3KC3 interaction.

\section{Discussion}

To the best of our knowledge, this is first report to demonstrate that the anti-inflammatory effect of AS acts via inhibition of autophagic activation by blocking the TRAF6-Beclin1PI3KC3 pathway and decreasing K63-linked ubiquitination of Beclin1, which interrupts the formation of the Beclin1-PI3KC3 core complex.

Sepsis is an infection-initiated systemic inflammation that occurs with high incidence in the critically ill population $[21,22]$. The induced inflammatory response usually becomes severe and uncontrolled in a relatively short time, leading to acute organ dysfunction and other systemic disorders [23]. Despite progress in medical care and treatment, the morbidity of sepsis increases annually and the mortality remains remarkably high [24]. Sepsis is still widely regarded as a major medical problem that induces a prevalent public health burden to society and challenges clinicians and other healthcare staff worldwide. To date, several pathogen-associated molecular patterns (PAMPs), such as LPS, CpG DNA, and peptidoglycans, have been identified as virulence factors for sepsis [25]. Among these PAMPs, LPS plays the most critical role in sepsis and subsequent organ dysfunction and death [26]. LPS is recognized by TLR4 on the membrane of innate immune system cells, such as monocytes and macrophages, and then triggers MyD88-dependent or -independent activation of TRAF6, leading to NF- $\kappa \mathrm{B}$ activation and downstream pro-inflammatory cytokine release [5]. 


\section{Cellular Physiology Cell Physiol Biochem 2018;47:475-488 and Biochemistry Published online: May 24, $2018 \quad \begin{aligned} & \text { DOI: 10.1159/000489982 } 2018 \text { The Author(s). Published by S. Karger AG, Basel } \\ & \text { www.karger.com/cpb }\end{aligned}$ \\ Kuang et al.: Artesunate Inhibits the TRAF6-Beclin1-PI3KC3 Pathway}

However, no drug targeting LPS or the LPS/TLR4-mediated pathway has entered clinical use. Therefore, it is important and urgent to investigate new drugs to treat sepsis.

TLR4 is not only the key receptor for LPS-mediated TRAF6-NF- $\kappa B$ pathway to release cytokines, but also is a sensor that participates in LPS-induced autophagy, and the two pathways share several key signaling molecules [6]. LPS induces macroautophagy by activating the PI3K-III complex, or aggrephagy through priming TRAF6-mediated recruitment of SQSTM1 [8], and autophagy regulates LPS-induced pro-inflammatory cytokines production [9]. Therefore, the autophagy pathway has become a new target to develop anti-sepsis agents. Autophagy inhibitors, such as 3-MA, wortmannin, and LY294002 targeting PI3KC3, showed notable anti-inflammatory effects in septic mice and in vitro [27]; however, their applications in sepsis or other inflammatory diseases are limited because of their cell toxicity. Therefore, it is necessary to develop new drugs to treat sepsis that regulate autophagy.

In our laboratory, AS was previously reported to possess anti-inflammation effects. AS can inhibit TLR4-mediated NF- $\kappa B$ activation, leading to decreased release of pro-inflammatory cytokines, such as TNF- $\alpha$ and IL-6 [10]. In the present study, we showed that AS could inhibit cytokines release from not only RAW264.7 cells, but also from primary mouse macrophages, including BMDMs and PMs, which supported the results of our previous report. Although AS was noted to inhibit the growth of MCF-7 and MDA-MB-231 breast cancer cells, which are related to autophagy [28], there has been no report of AS's anti-inflammatory effect being related to autophagy. We observed that AS's anti-inflammatory effect in autophagic activation was inhibited by 3-MA (which targets PI3KC3) and Baf (which targets lysosome acidification and blocks autophagic flux), which demonstrated that AS's effects are closely associated with autophagy.

TRAF6 is an unique member among TRAF family in that it also participates in IL-1 receptor (IL-1R)/TLR super family signaling through MyD88-dependent or -independent pathways, thereby playing an essential role in innate immune responses, including inflammation and autophagy [6]. TRAF6 catalyzes K63-linked ubiquitination of IL-1 receptor-associated kinase 1 (IRAK1) and self-ubiquitination, which leads to NF- $\kappa B$ activation and inflammation-related cytokines expression [25]. Meanwhile, TRAF6 also catalyzes K63-linked ubiquitination of Beclin1, a key component of the PI3K-III complex that initiates autophagosome formation [20]. Therefore, TRAF6 is a key factor in both the LPS/TLR4-mediated inflammation pathway and TLR4-triggered autophagy pathway via its E3 ligase activity [29]. In addition, TRAF6 initiates aggrephagy by recruiting certain autophagic receptors, including SQSTM1, HDAC6, and NDP52 [6]. We demonstrated that AS could decrease TRAF6 levels, which could be considered to result from the inhibition by AS of TIr4 mRNA expression and NF- $\kappa$ B activation, which was reported previously [10].

Beclin1 is a key regulator of autophagy [30]. As a substrate of TRAF6, Beclin1 is a component of the PI3K-III complex and serves as a scaffolding protein to recruit PI3KC3, forming the PI3K-III complex, which is essential for formation of the phagophore in the autophagy machinery [31]. Therefore, K63-linked ubiquitination of Beclin1 plays a very important role during autophagosome formation, and interfering with this function would disrupt the autophagy machinery. In the present study, AS was shown to decrease Beclin1 and PI3KC3 levels, which could also be considered to result from the previously reported inhibition by AS of TLR4-NF- $\kappa B$ [10].

As described above, K63-linked ubiquitination of Beclin1 by TRAF6 is critical for TLR4triggered autophagy in macrophages. In the present study, AS was found to not only decrease TRAF6, Beclin1, and PI3KC3 levels, but also significantly inhibited K63-linked ubiquitination of Beclin1 induced by LPS. However, AS had no influence on the total ubiquitination of Beclin1. When an equal amount of Beclin1 was used, Beclin1 (as the substrate) could pulldown an equal amount of TRAF6 (enzyme); but when equal amount of TRAF6 was used, TRAF6 pulled down a lower amount of Beclin1. These results demonstrated when there was equal substrate, an equal amount of enzyme was induced to bind; however, when there was an equal amount of enzyme, enzyme could not induce an equal amount of substrate to 


\section{Cellular Physiology Cell Physiol Biochem 2018;47:475-488 \begin{tabular}{l|l} 
DOI: 10.1159/000489982 & O 2018 The Author(s). Published by S. Karger AG, Basel \\
www.karger.com/cpb
\end{tabular} \\ Kuang et al.: Artesunate Inhibits the TRAF6-Beclin1-PI3KC3 Pathway}

bind, which suggested that AS acts on the enzyme to interrupt its binding with the substrate, indirectly demonstrating AS could inhibit the E3 ligase activity of TRAF6. This deduction was confirmed by detecting the levels of K63-linked ubiquitination of Beclin1. However, the mechanism by which AS inhibits E3 the ligase activity of TRAF6 requires further investigation.

In summary, AS could inhibit the LPS-induced release of cytokines from macrophages by inhibiting the activation of autophagy. This effect is closely related to blockade of the TRAF6-Beclin1-PI3KC3 pathway via decreasing K63-linked ubiquitination of Beclin1, which interrupts the formation of Beclin1-PI3KC3 core complex of the PI3K-III complex. Our findings reveal the mechanism of AS's anti-inflammatory effect and lays the foundation for future studies of targeted sepsis treatment.

\section{Acknowledgements}

This work was supported by the National Natural Science Foundation of China [grant number 81673495] and the Special Basic Science and Cutting-edge Research Project of Chongqing [grant number cstc2015jcyjBX0049].

\section{Disclosure Statement}

The authors declare that they have no conflict of interests.

\section{References}

1 Reinhart K, Daniels R, Kissoon N, Machado FR, Schachter RD, Finfer S: Recognizing Sepsis as a Global Health Priority - A WHO Resolution. New Engl J Med 2017;377:414-417.

-2 McPherson D, Griffiths C, Williams M, Baker A, Klodawski E, Jacobson B, Donaldson L: Sepsis-associated mortality in England: an analysis of multiple cause of death data from 2001 to 2010 BMJ Open 2013;3

- Ramachandran G: Gram-positive and gram-negative bacterial toxins in sepsis: a brief review. Virulence 2014;5:213-218

4 Tsujimoto H, Ono S, Efron PA, Scumpia PO, Moldawer LL, Mochizuki H: Role of Toll-like receptors in the development of sepsis. Shock 2008;29:315-321.

5 Rosadini CV, Kagan JC: Early innate immune responses to bacterial LPS. Curr opin immunol 2017;44:14-19.

6 Into T, Inomata M, Takayama E, Takigawa T: Autophagy in regulation of Toll-like receptor signaling. Cell signal 2012;24:1150-1162.

7 Abrahamsen H, Stenmark H, Platta HW: Ubiquitination and phosphorylation of Beclin 1 and its binding partners: Tuning class III phosphatidylinositol 3-kinase activity and tumor suppression. FEBS lett 2012;586:1584-1591.

8 Fujita K, Maeda D, Xiao Q, Srinivasula SM: Nrf2-mediated induction of p62 controls Toll-like receptor4-driven aggresome-like induced structure formation and autophagic degradation. P Natl Acad Sci USA 2011;108:1427-1432.

-9 Qian M, Fang X, Wang X: Autophagy and inflammation. Clin Transl Med 2017;6:24.

10 Li B, Zhang R, Li J, Zhang L, Ding G, Luo P, He S, Dong Y, Jiang W, Lu Y, Cao H, Zheng J, Zhou H: Antimalarial artesunate protects sepsis model mice against heat-killed Escherichia coli challenge by decreasing TLR4, TLR9 mRNA expressions and transcription factor NF-kappa B activation. Int Immunopharmacol 2008;8:379-389.

11 Li B, Yu M, Pan X, Ren C, Peng W, Li X, Jiang W, Zheng J, Zhou H: Artesunate reduces serum lipopolysaccharide in cecal ligation/puncture mice via enhanced LPS internalization by macrophages through increased mRNA expression of scavenger receptors. Int J Mol Sci 2014;15:1143-1161.

12 Pan X, Yue J, Ding G, Li B, Liu X, Zheng X, Yu M, Li J, Jiang W, Wu C, Zheng J, Zhou H: Leucine-rich repeat 11 of Toll-like receptor 9 can tightly bind to CpG-containing oligodeoxynucleotides, and the positively charged residues are critical for the high affinity. J Biol Chem 2012;287:30596-30609. 


\section{Cellular Physiology Cell Physiol Biochem 2018;47:475-488

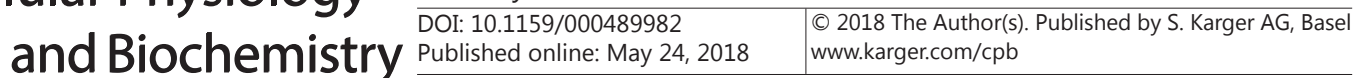 \\ Kuang et al.: Artesunate Inhibits the TRAF6-Beclin1-PI3KC3 Pathway}

13 Liu X, Wang N, Zhu Y, Yang Y, Chen X, Chen Q Zhou H, Zheng J: Extracellular calcium influx promotes antibacterial autophagy in Escherichia coli infected murine macrophages via CaMKKbeta dependent activation of ERK1/2, AMPK and Fox01. Biochem Bioph Res Co 2016;469:639-645.

14 Zhou J, Li G, Zheng Y, Shen HM, Hu X, Ming QL, Huang C, Li P, Gao N: A novel autophagy/mitophagy inhibitor liensinine sensitizes breast cancer cells to chemotherapy through DNM1L-mediated mitochondrial fission. Autophagy 2015;11:1259-1279.

-15 Popko K, Gorska E, Stelmaszczyk-Emmel A, Plywaczewski R, Stoklosa A, Gorecka D, Pyrzak B, Demkow U: Proinflammatory cytokines Il-6 and TNF-alpha and the development of inflammation in obese subjects. Eur J Med Res 2010;15:S120-122.

-16 Waltz P, Carchman EH, Young AC, Rao J, Rosengart MR, Kaczorowski D, Zuckerbraun BS: Lipopolysaccaride induces autophagic signaling in macrophages via a TLR4, heme oxygenase-1 dependent pathway. Autophagy 2011;7:315-320.

17 Wesselborg S, Stork B: Autophagy signal transduction by ATG proteins: from hierarchies to networks. Cell Mol Life Sci: CMLS 2015;72:4721-4757.

18 Schaaf MB, Keulers TG, Vooijs MA, Rouschop KM: LC3/GABARAP family proteins: autophagy-(un)related functions. FASEB J 2016;30:3961-3978.

19 Bosmann M, Ward PA: The inflammatory response in sepsis. Trends Immunol 2013;34:129-136.

20 Shi CS, Kehrl JH: TRAF6 and A20 regulate lysine 63-linked ubiquitination of Beclin-1 to control TLR4induced autophagy. Sci Signal 2010;3:ra42.

-21 Stearns-Kurosawa DJ, Osuchowski MF, Valentine C, Kurosawa S, Remick DG: The pathogenesis of sepsis. Annu Rev Pathol 2011;6:19-48.

22 Lolis E, Bucala R: Therapeutic approaches to innate immunity: severe sepsis and septic shock. Nat Rev Drug Discov 2003;2:635-645.

23 Lakshmikanth CL, Jacob SP, Chaithra VH, de Castro-Faria-Neto HC, Marathe GK: Sepsis: in search of cure. Inflamm Res 2016;65:587-602.

24 Walkey AJ, Lagu T, Lindenauer PK: Trends in sepsis and infection sources in the United States. A population-based study. Annu Am Thorac Soc 2015;12:216-220.

25 Brubaker SW, Bonham KS, Zanoni I, Kagan JC: Innate immune pattern recognition: a cell biological perspective. Annu Rev Immunol 2015;33:257-290.

26 Cavaillon JM: Exotoxins and endotoxins: Inducers of inflammatory cytokines. Toxicon 2017

-27 Deretic V, Saitoh T, Akira S: Autophagy in infection, inflammation and immunity. Nat Rev Immunol 2013;13:722-737.

28 Chen K, Shou LM, Lin F, Duan WM, Wu MY, Xie X, Xie YF, Li W, Tao M: Artesunate induces G2/M cell cycle arrest through autophagy induction in breast cancer cells. Anti-Cancer Drug 2014;25:652-662.

29 Kawai T, Akira S: TLR signaling. Cell Death Differ 2006;13:816-825.

30 Wirawan E, Lippens S, Vanden Berghe T, Romagnoli A, Fimia GM, Piacentini M, Vandenabeele P: Beclin1: a role in membrane dynamics and beyond. Autophagy 2012;8:6-17.

-31 Sriram SM, Han DH, Kim ST: Partners in crime: ubiquitin-mediated degradation and autophagy. Sci signal 2011;4:jc4. 